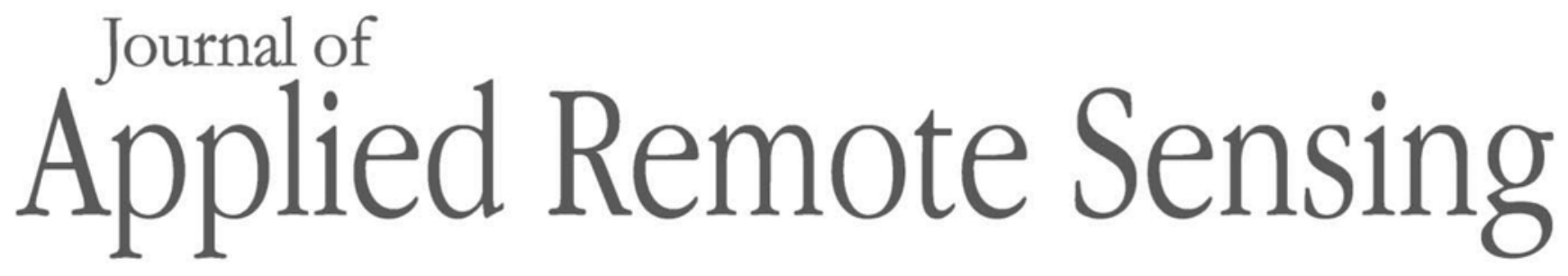

RemoteSensing.SPIEDigitalLibrary.org

\title{
Fully automated laboratory and field-portable goniometer used for performing accurate and precise multiangular reflectance measurements
}

Justin D. Harms

Charles M. Bachmann

Brittany L. Ambeau

Jason W. Faulring

Andres J. Ruiz Torres

Gregory Badura

Emily Myers

Justin D. Harms, Charles M. Bachmann, Brittany L. Ambeau, Jason W. Faulring, Andres J. Ruiz Torres, Gregory Badura, Emily Myers, "Fully automated laboratory and field-portable goniometer used for 


\title{
Fully automated laboratory and field-portable goniometer used for performing accurate and precise multiangular reflectance measurements
}

\author{
Justin D. Harms, ${ }^{\text {a,* }}$ Charles M. Bachmann, ${ }^{\text {a }}$ Brittany L. Ambeau, ${ }^{\text {a }}$ \\ Jason W. Faulring, ${ }^{b}$ Andres J. Ruiz Torres, ${ }^{a}$ Gregory Badura, ${ }^{\text {a }}$ and \\ Emily Myers ${ }^{a}$ \\ ${ }^{a}$ Rochester Institute of Technology, Chester F. Carlson Center for Imaging Science, \\ Rochester, New York, United States \\ ${ }^{\mathrm{b}}$ AppliedLogix, LLC, Fairport, New York, United States
}

\begin{abstract}
Field-portable goniometers are created for a wide variety of applications. Many of these applications require specific types of instruments and measurement schemes and must operate in challenging environments. Therefore, designs are based on the requirements that are specific to the application. We present a field-portable goniometer that was designed for measuring the hemispherical-conical reflectance factor (HCRF) of various soils and low-growing vegetation in austere coastal and desert environments and biconical reflectance factors in laboratory settings. Unlike some goniometers, this system features a requirement for "target-plane tracking" to ensure that measurements can be collected on sloped surfaces, without compromising angular accuracy. The system also features a second upward-looking spectrometer to measure the spatially dependent incoming illumination, an integrated software package to provide full automation, an automated leveling system to ensure a standard frame of reference, a design that minimizes the obscuration due to self-shading to measure the opposition effect, and the ability to record a digital elevation model of the target region. This fully automated and highly mobile system obtains accurate and precise measurements of HCRF in a wide variety of terrain and in less time than most other systems while not sacrificing consistency or repeatability in laboratory environments. () The Authors. Published by SPIE under a Creative Commons Attribution 3.0 Unported License. Distribution or reproduction of this work in whole or in part requires full attribution of the original publication, including its DOI. [DOI: 10.1117/1.JRS.11.046014]
\end{abstract}

Keywords: goniometer of the Rochester Institute of Technology-two; bidirectional reflectance distribution function; hemispherical-conical reflectance factor; biconical reflectance factor; digital elevation model; dual view; full automation; target-plane tracking.

Paper 170214 received Mar. 9, 2017; accepted for publication Nov. 7, 2017; published online Nov. 24, 2017.

\section{Introduction}

The goniometer of the Rochester Institute of Technology-two (GRIT-T) is a second-generation goniometric system that was designed and built in the Chester F. Carlson Center for Imaging Science at the Rochester Institute of Technology (RIT). Similar to the first-generation system, the goniometer of the Rochester Institute of Technology (GRIT), ${ }^{1}$ the GRIT-T collects reflected radiance on a spherical grid in both laboratory and field settings. However, in a field setting, the GRIT-T collects simultaneous incoming and reflected radiance. The primary purpose of the system is to estimate the biconical reflectance factor (BCRF) and hemispherical-conical reflectance factor $(\mathrm{HCRF})^{2-4}$ of various soils and short vegetation to better understand the limits of existing radiative transfer models, to validate modified radiative transfer models, ${ }^{5,6}$ and to aid in the vicarious calibration of remote sensing platforms. ${ }^{7}$ Reflectance factors are calculated by comparing the radiance of a target surface to that of a Lambertian standard reference. ${ }^{2-4}$ Specifically,

*Address all correspondence to: Justin D. Harms, E-mail: jdh1069@rit.edu 
a reflectance factor RF is estimated from the ratio of the radiance $L_{r}$ reflected from the surface to the radiance $L_{\text {ref }}$ reflected from a Spectralon reference panel. Since white reference panels, such as Spectralon ${ }^{\mathrm{TM}}$ reference panels, do not show ideal Lambertian reflectance behavior, the radiance $L_{\text {ref }}$ has to be corrected by a panel calibration coefficient $C_{\text {ref }}$. Thus ${ }^{8}$

$$
\mathrm{RF}\left(\theta_{i}, \phi_{i}, \theta_{r}, \phi_{r}\right)=\frac{L_{r}\left(\theta_{i}, \phi_{i}, \theta_{r}, \phi_{r}\right)}{L_{\mathrm{ref}}\left(\theta_{i}, \phi_{i}, \theta_{r}, \phi_{r}\right)} \cdot C_{\mathrm{ref}}\left(\theta_{i}, \phi_{i}, \theta_{r}, \phi_{r}\right),
$$

where $\theta_{i}$ and $\phi_{i}$ are the incident zenith and azimuth angles, respectively, and $\theta_{r}$ and $\phi_{r}$ are the zenith and azimuth angles of observation, respectively. The term "biconical" in BCRF refers to the fact that the physical extent of both the sensor and the source of illumination are not idealized differential quantities but, though small, have a finite size. ${ }^{2-4,6}$ BCRF usually describes the type of measurement undertaken within a laboratory setting with a single light source and sensor. In contrast in outdoor settings, where the light is from both a directional source (the Sun) and from a skylight, the term HCRF is used. ${ }^{2-4,6}$

To accomplish these types of measurements, the GRIT-T incorporates many features into one compact design. While some of the features were leveraged from other systems, such as the dualview capability and the full automation, other features were added to satisfy the scientific requirements of our research group. These new features include target-plane tracking, automated system leveling, minimized obscuration due to self-shading, digital elevation models (DEMs), and a number of other features to improve accuracy, mobility, and rigidity. After two years of design and development, the GRIT-T was tested in the laboratory at RIT and in a field experiment in the Nevada desert in June 2016. In this paper, we expand on a brief conference publication written during the development of the instrument ${ }^{9}$ describing in full detail the features of the GRIT-T and the initial results from both laboratory and field experiments. Figure 1 provides an overview of the GRIT-T and labels the main components of the system.

\section{Background}

As previously mentioned, the GRIT-T is the second-generation goniometric system created by RIT. The first-generation system, GRIT, was a prototype developed by our research group to measure BCRF in the laboratory and HCRF in the field and to further our research in the theory and application of radiative transfer models. ${ }^{1}$ The lessons learned from operating the GRIT for two years greatly impacted the design and engineering of the second-generation system. Since the GRIT had been operated in countless laboratory experiments and multiple field experiments, our research group was able to identify the improvements necessary to achieve both better accuracy and precision as well as improved mobility. These insights quickly became the tenets of the GRIT-T design and were combined with attractive features that were found in other published systems, e.g., the dual field-of-view (FoV) FIGOS, the GOPHER, the automated spectrogoniometer, the FIGIFIGO, and the University of Twente goniometer. ${ }^{6,10-13}$ These systems, and the GRIT, ${ }^{1}$ provided inspiration for the GRIT-T design and had a dramatic impact on the overall capabilities and features implemented in the GRIT-T.

\section{System Design}

The GRIT-T incorporates many features - some found in other systems and some that are new to the field-into one compact design that can measure the multiangular reflectance of a target in both a laboratory and field setting. In this paper, the features of the system will be discussed. These features include the capability to monitor downwelling radiance and reflected radiance simultaneously, a fully automated operating scheme, target-plane tracking to minimize wandering spot errors, minimized obscuration of reflectance measurements to mitigate self-shading, and the capability to measure a DEM of the target surface. Because of these features, during BCRF and HCRF measurements, the angular position accuracy of the system is \pm 0.2 deg in both sensor azimuth and zenith orientations. 


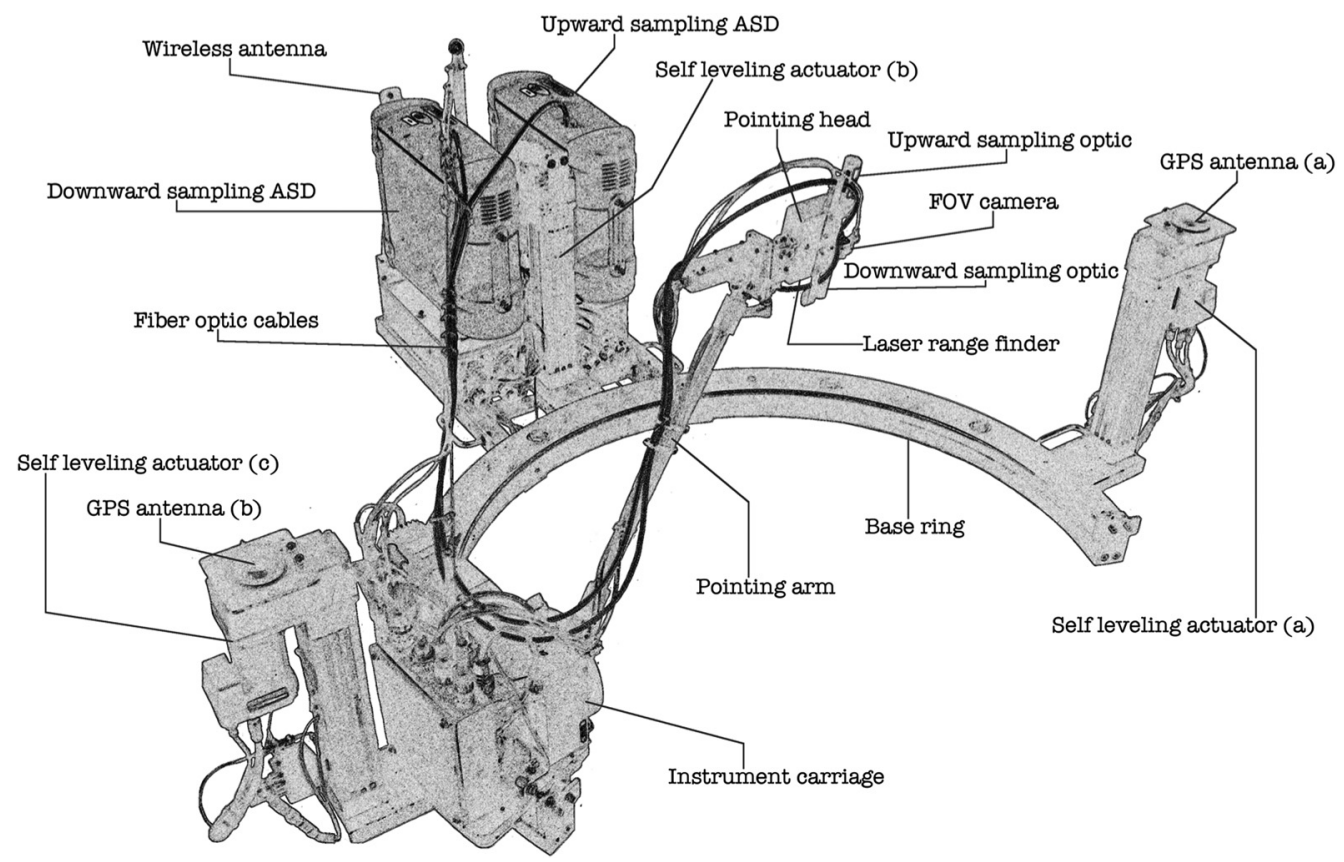

Fig. 1 The hyperspectral gonimeter system, GRIT-T, with its main components labeled. GRIT-T hosts two on-board ASD, Inc. FR 4 spectrometers: one measures the incoming radiance onto the target while the other measures the scattered radiance from the surface. The $\mathrm{C}$-shaped ring and the rotating arm/head of the instrument allow positioning of the sensor over an azimuth range of 0 deg to $180 \mathrm{deg}$ and zenith range of $-70 \mathrm{deg}$ to $+70 \mathrm{deg}$. The design also ensures minimal selfshading onto the measurement surface. The design includes self-leveling actuators, which enable the system to maintain a standard reference frame throughout measurement. The dual-antenna the global positioning system (GPS) IMU provides geolocation information. An on-board laser range finder estimates distance to the surface at nadir to allow tracking of the same location with the counter-rotating pointing head and also measures a DEM of the surface in a separate scan mode. A miniature camera on the pointing head records the FoV. Not shown: shield painted in Krylon flat black that is placed in front of the spectrometers to prevent secondary scatter into the sampling fore-optic from the instrument housing (the shield can be seen, for example, in Figs. 2 and 5).

The GRIT-T is capable of positioning the sensor fore-optic over an azimuth range of 0 deg to $180 \mathrm{deg}$, and a zenith range from $-70 \mathrm{deg}$ to $+70 \mathrm{deg}$, to sample the reflected radiance in the hemisphere above a target surface. The narrow profile of the instrument's arm and the ability to rotate the instrument's head result in a significant decrease in the amount of self-shading onto the target surface during hemispherical measurements when compared to our laboratory's previous goniometer, GRIT. In the previous goniometer design of GRIT, a broad azimuthal swath of $\pm 15 \mathrm{deg}$ from the backscatter region of the principal plane was completely shadowed by the arc of the goniometer. ${ }^{1}$ In contrast, the GRIT-T is designed so that only regions within phase angles of $\sim 5$ deg from the illumination source orientation are partially shadowed by the instrument profile. In addition to an increased achievable range for directional reflectance measurements, this design allows the system to consecutively measure the forward and backward segments of the solar principal plane with minimal time in between traversals. To implement this sampling technique, the GRIT-T uses a carriage to rotate the sensor along a C-shaped (i.e., $220 \mathrm{deg}$ ) ring. As the carriage moves from one position in the azimuth range to the next, a pointing arm and pointing head rotate the sensor along the zenith range. Figure 2 shows the GRIT-T as it operates in a laboratory setting.

\subsection{Dual-View Capability}

In a field setting, it is very difficult to decouple the effect of the sky illumination from that of the solar illumination in the observed target reflectance across the full range of the solar irradiance spectrum. Many researchers have relied on atmospheric models to aid in the understanding 


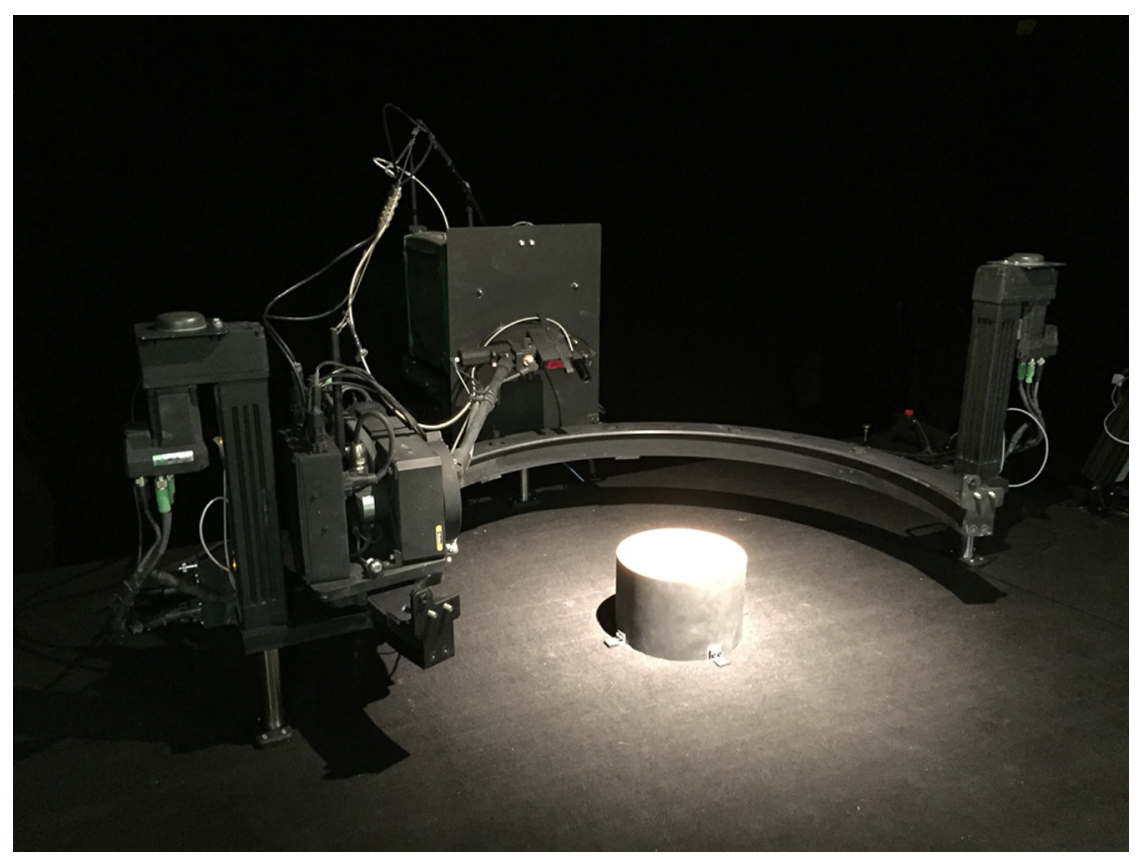

Fig. 2 The GRIT-T instrument being operated in a dark room laboratory setting. The walls have been covered with black lab-grade felt in order to minimize diffuse scattering onto the sample of interest. In the center of the C-shaped ring of GRIT-T is a sample holder filled with sandy sediment that is being used in a laboratory study.

the impact of the sky illumination. However, this approach can be prone to modeling errors if the model input parameters are not well characterized. ${ }^{14,15}$ Typically, these parameters are determined by using a locally launched radiosonde. However, in many situations, this technique may also be inadequate if the sky conditions are rapidly changing. It is for these reasons that many researchers in the remote sensing community prefer to coincidently measure the downwelling illumination with the target reflectance and incorporate these additional measurements into their postprocessing routines to better compensate for temporal changes in illumination that occur throughout the course of a scan. , $^{6,10,16}$

While the downwelling illumination measurements are useful for removing the temporal changes, these measurements do not compensate for the spatially dependent incoming illumination from nearby objects (i.e., adjacency effects). Specifically, when measuring an HCRF, it is necessary to understand the angular distribution of the incoming radiance. It is for this reason that the GRIT-T incorporates an upward-looking, dual-view capability, which allows for the measurement of the spatially dependent incoming illumination, similar to the dual FoV FIGOS goniometer. ${ }^{10}$

Both of the on-board spectrometers, one upward-looking and one downward-looking along the same optical axis, are Analytical Spectral Devices (ASD), Inc. FieldSpec 4 Hi-Res spectroradiometers. ${ }^{17}$ By using two spectrometers with the same make and model, we reduce the likelihood of interpolation errors when comparing one grid to another. These spectrometers are equipped with three detectors that cover the spectral range of 350 to $2500 \mathrm{~nm}$ : a silicon array for the visible and near-infrared (VNIR) spectral range of 350 to $1000 \mathrm{~nm}$, a cooled InGaAs photodiode detector for the short-wave infrared (SWIR) range of 1001 to $1800 \mathrm{~nm}$, and a cooled InGaAs photodiode detector for the SWIR spectral range of 1801 to $2500 \mathrm{~nm}$. These systems are annually calibrated by the manufacturer to maintain both spectral and radiometric accuracy using National Institute of Standards and Technology (NIST)-traceable standards. In the spectral domain, the annual calibrations are certified to provide resolutions of $3 \mathrm{~nm}$ in the VNIR and $8 \mathrm{~nm}$ in the SWIR. In addition, these systems are able to achieve wavelength accuracies of $0.5 \mathrm{~nm}$ and wavelength reproducibility to within $0.1 \mathrm{~nm} .{ }^{17}$ The annual radiometric calibration for the spectrometers results in an absolute radiance accuracy typically $\leq 2.5 \%$ between 655 and $2400 \mathrm{~nm}$ with uncertainty being greater at the ends of the wavelength range (typically $\leq 3.6 \%$ 


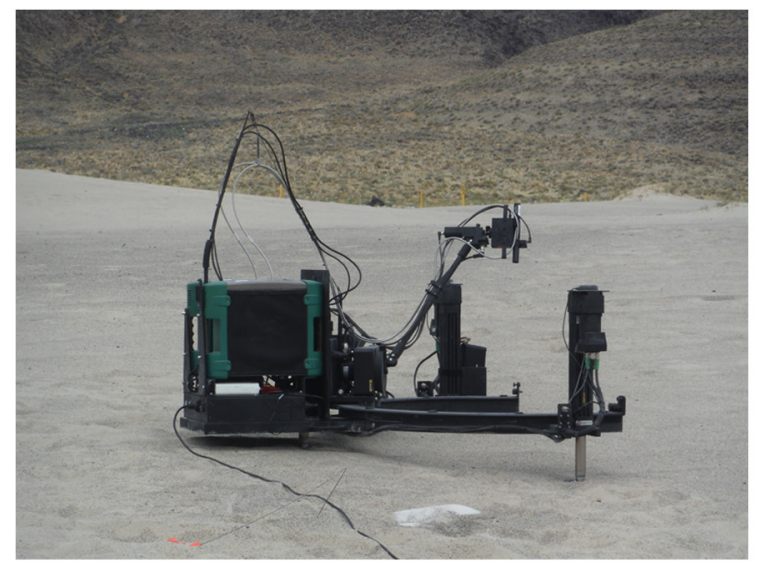

(a)

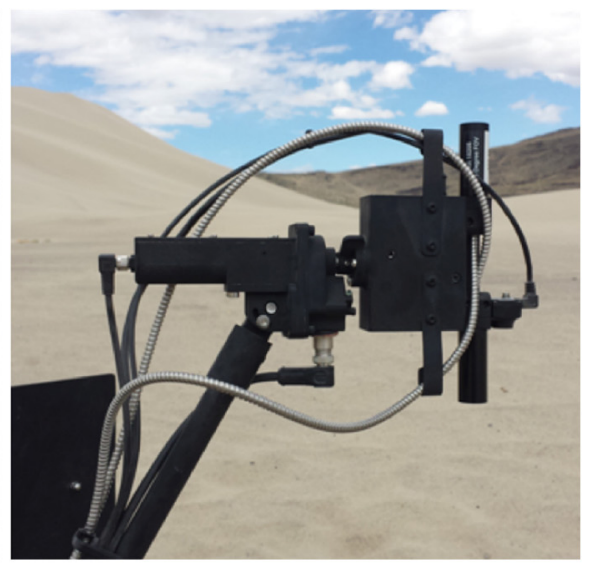

(b)

Fig. 3 (a) Image of GRIT-T with the two ASD fiber optic cables that are guided above the shield and along the pointing arm. Once guided, the two ASD fiber optic cables are inserted into the GRIT-T pointing head, either upward or downward, and two ASD fore-optics are attached. (b) The GRIT-T pointing head with the two ASD fore-optics and ASD fiber optic cables.

absolute accuracy at $350 \mathrm{~nm}$ ). This calibration also indicates an uncertainty of $0.5 \%$ in reflectance from 350 to $2000 \mathrm{~nm}$, with the uncertainty rising to $2 \%$ at $2400 \mathrm{~nm}$. Each spectrometer can be fitted with multiple fore-optic attachments including: $1 \mathrm{deg}, 3 \mathrm{deg}, 5 \mathrm{deg}$, and $8 \mathrm{deg}$. The fiber optic with no fore-optic attachment has a 25 -deg FoV. ${ }^{17}$ Normally, the 5-deg fore-optic attachment will be used on the downward-looking spectrometer, and an 8-deg fore-optic attachment will be used on the upward-looking spectrometer. This is done with the intent of monitoring a small, homogenous region on the ground while simultaneously capturing the sky conditions over a somewhat broader angular extent.

By designing the system so that the downward-looking spectrometer is aligned along the same optical axis as the upward-looking spectrometer, the GRIT-T measures the incoming radiance onto the target over the entire spherical grid while simultaneously characterizing the angular dependence of the scattered radiance from the surface. A third spectrometer is then used to ensure that any temporal changes in illumination are measured for compensation in the postprocessing routines; typically, this third spectrometer will monitor the reflected radiance from a Spectralon ${ }^{\mathrm{TM}}$ calibration panel or monitor the downwelling radiance onto the surface by means of a cosine collector fore-optic attachment. ${ }^{16}$ This third spectrometer is located at a stationary location near the GRIT-T. Due to its lightweight frame design, the GRIT-T is able to add this dual-view capability without dramatically reducing the mobility of the system, and the software is able to integrate the third spectrometer into the overall control scheme since the control of all on-board devices operates on a separate internal network. Figure 3 shows the placement of the spectrometers along with the fiber optic cables and the design of the fore-optic pointing head. In addition to using a third spectrometer to monitor temporal changes in illumination conditions, we determined through laboratory studies that frequent measurements by the upward-looking and downward-looking spectrometers at the nadir orientation during the execution of a hemispherical scan pattern also can be used to monitor and compensate for variations in overall radiance levels that may occur over time. Using all of this information, we have developed postprocessing routines to ensure that all reflected radiance measurements have been corrected for variations in downwelling radiance levels over the course of the scan.

\subsection{Full Automation}

One of the main limitations of the first-generation system GRIT $^{1}$ was that of operator fatigue resulting from the manual operation of the instrument in harsh environments. The device required a considerable amount of user input to operate with the significant potential for user error from lapses in operator attention. For this reason, the GRIT-T was designed to be fully autonomous during a scan. To perform a GRIT-T scan, the user sets the scan pattern in the graphical user interface (GUI) provided by the integrated GRIT-T software. Once the 


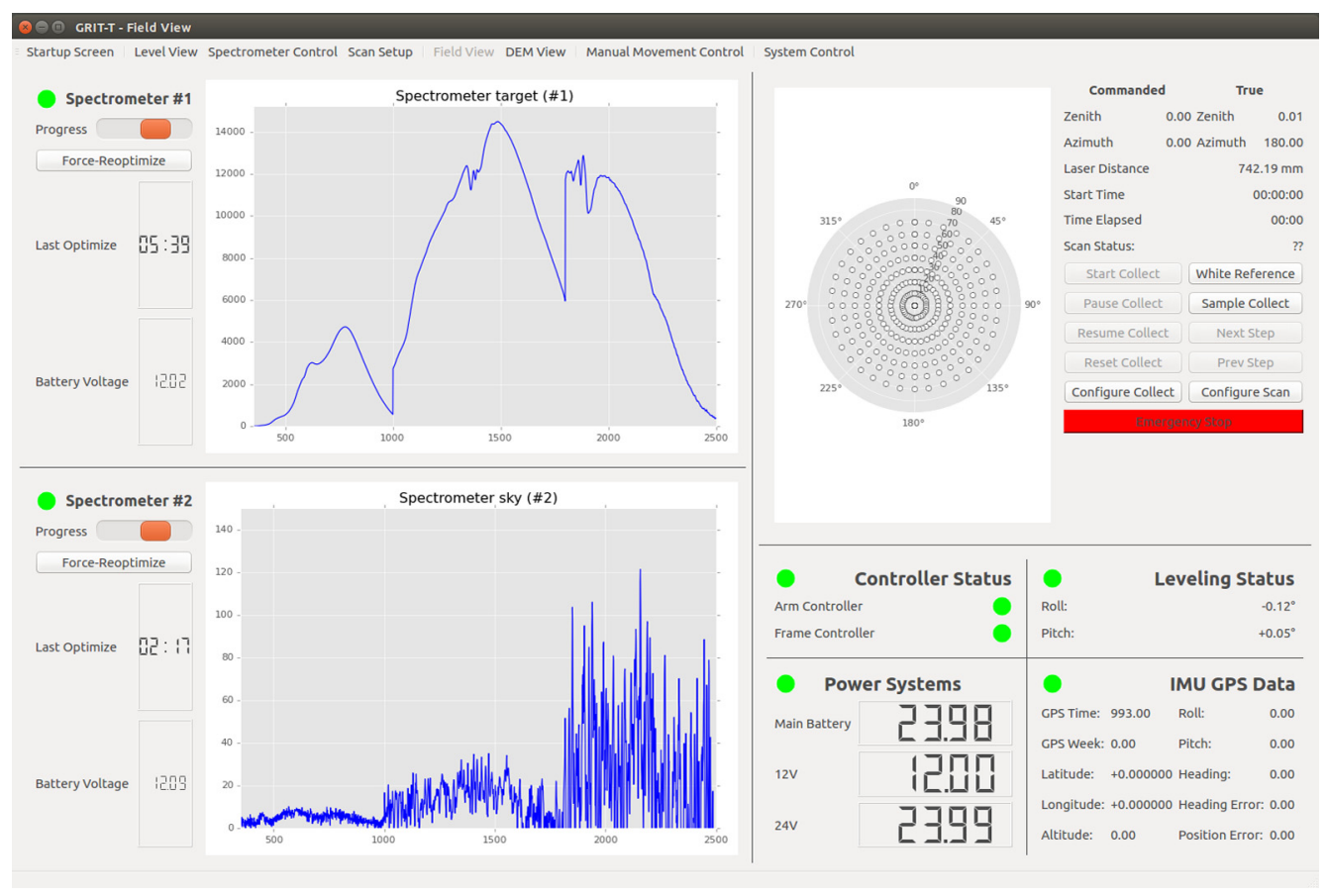

Fig. 4 Diagnostic information during measurement observed through the GUI of the goniometer system. The interface shows the spectra in digital counts for the upward- and downward-looking spectrometers. It also monitors the progress of the generated scan pattern, the power supply, and the GPS data provided by the IMU. Shown: field view tab, one of 8 command, control, and monitoring tabs available with the GUI.

user starts the scan with the GUI, the system moves the sensor to the predefined positions on the spherical grid and saves the data from the spectrometers to an on-board computer. In addition to the data saved from the spectrometers, the system saves the corresponding time-stamped data from the other on-board devices. These on-board devices include the VectorNav VN-300 inertial measurement unit (IMU) and GPS, which provides the heading information; the NIST-Traceable Rieker Precision Inclinometer, which provides the leveling information; and the encoders, which provide the pointing information relative to the system frame of reference provided by the onboard VectorNav IMU/GPS system. As the scan progresses, the user can monitor the status of the scan, the health of subsystems, the real-time spectra from the spectrometers, and the positioning information from the on-board GPS-enabled IMU. The diagnostic information available on the field view tab of the GUI is shown in Fig. 4.

The interface in Fig. 4 provides the user with a comprehensive status of the measurement/ scan being performed. In this specific interface, the user has the ability to begin, pause, or end the measurement at anytime. It can monitor the progress of the generated scan pattern, the operation of the embedded computers, the roll and pitch of the frame, the power supply, and the health of the GPS data provided by the IMU. Furthermore, this interface allows the user to monitor the real-time spectra in digital counts from the upward- and downward-looking spectrometers, the time since last optimization and the battery voltage of the spectrometers. The "status" indicators change from green to red to warn the user if any errors are being sent from the spectrometers or the subsystems.

\subsection{Target-Plane Tracking}

One of the key goals of the GRIT-T design was to ensure that the view angles requested by the user were achieved without effects due to parallax. Parallax error occurs when a goniometric system uses a measurement plane that is predefined and the measured scattering surface differs in height from the predefined measurement plane of the goniometer. To avoid this, the GRIT-T design implements "target-plane tracking," which is defined to be a measurement that ensures 
that the center of the FoV on the target surface remains the same regardless of changes in view angle or sensor distance from the surface. In order to achieve this stringent goal, GRIT-T utilizes a pointing arm, a pointing head, and a laser range finder that is accurate to within $\pm 2 \mathrm{~mm}$ and repeatable to within $\pm 0.5 \mathrm{~mm}$. Before performing a scan, the user inputs the parameters of the scan pattern in the GUI. Then, the system is commanded to measure the distance to the target at nadir using the laser range finder. Using this information in combination with the known geometry of the instrument frame and components, the system calculates motor movements required for the pointing arm and the pointing head, which both rotate to achieve the minimum parallax error for the commanded view angle. As previously mentioned, the pointing arm was designed to move the sensor from $-70 \mathrm{deg}$ to $+70 \mathrm{deg}$ in zenith. The rotating sensor head was designed to achieve the ability to aim the sensor at the same spot in the center of the azimuthal ring for every possible sensor viewing orientation. Thus, the pointing arm performs the large zenith movements, and the pointing head performs the small counter-rotated zenith offsets that ensure accurate tracking of the same spot on the target. This technique ensures that the GRIT-T can achieve the requested view angles with respect to the target without the undesired effect of wandering spot errors during measurements of the surface radiance. In addition, based on feedback from the on-board Rieker Precision Inclinometer, GRIT-T levels itself using an embedded control algorithm, which automatically adjusts the three system actuators to ensure that a standard frame of reference, is achieved during scans. No matter what height the GRIT-T ring is above the target surface, the measurement plane is always adjusted to the target surface using the recorded height from the laser range finder when at nadir. GRIT-T then automatically calculates the rotations of the pointing arm and point head needed to ensure tracking of the same point on the ground, as described above.

An example illustrating the simultaneously rotating sensor pointing head and arm appears in Fig. 5. Here, the GRIT-T is operating in a field setting and measuring the radiance from a sediment surface at a 70-deg zenith angle with respect to the target. Tests performed in laboratory settings show that the final system achieves the goal of tracking the center point of the azimuthal ring to within $10 \mathrm{~mm}$, even at the most extreme operating distances. During alignment of the GRIT-T pointing, we used nominal target distances of 632 and $732 \mathrm{~mm}$ obtained at nadir from the laser range finder, as these distances bound the range of most of the typical operating distances used in field and laboratory settings. To perform alignment, the downward-looking spectrometer and associated fore-optic were replaced with an optical alignment laser. The laser was

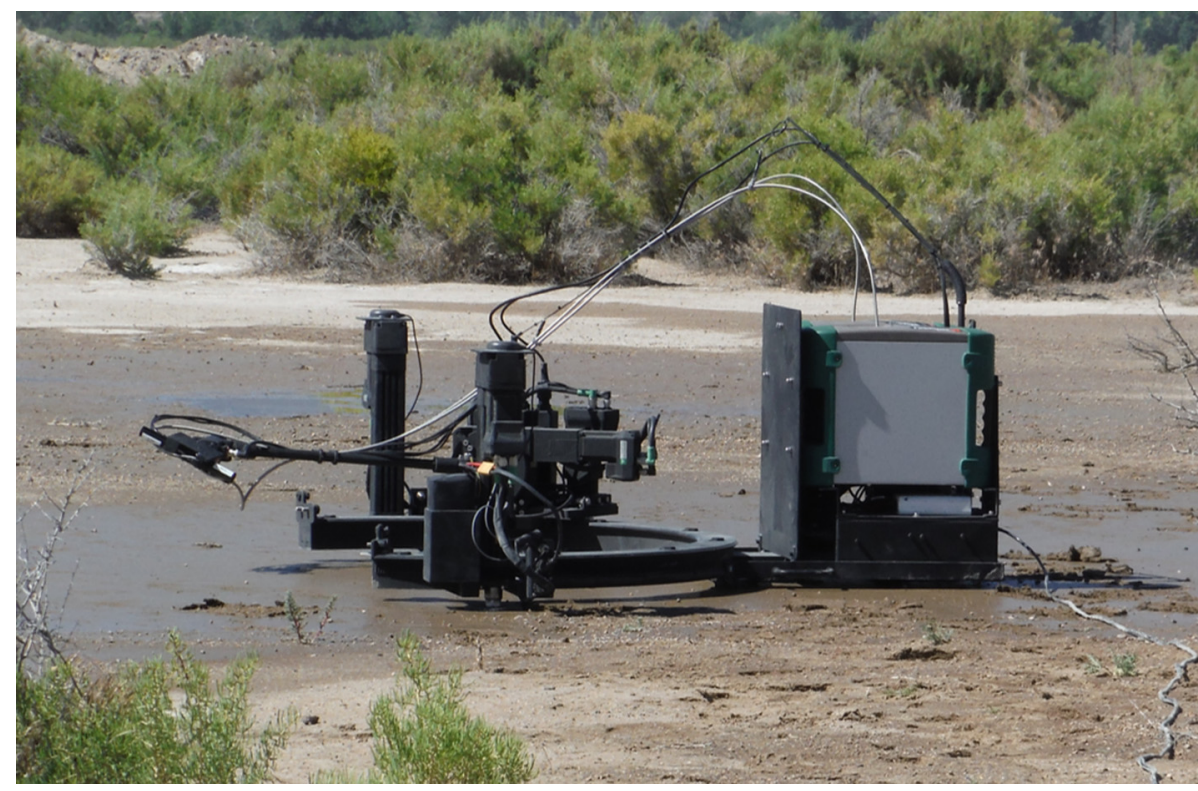

Fig. 5 The GRIT-T as it measures a "true" 70-deg zenith angle with respect to the target. Here, the GRIT-T pointing head is counter-rotated to ensure that the same spot on the target is measured at each view angle position. The magnitude of this counter-rotation is determined for every zenith view angle. 
optically aligned with a custom-designed holder, and the center of the ground instantaneous FoV was marked on leveled grid paper. The system then performed a hemispherical measurement sequence, in which the laser was positioned azimuthally in 45-deg intervals over a range from 0 deg to $360 \mathrm{deg}$. At each azimuthal orientation, the laser was oriented at zenith angles of $0 \mathrm{deg}$ and $65 \mathrm{deg}$. At each of these orientations of the laser, the center of the optically aligned laser spot on the leveled grid paper was recorded for later analysis. Figure 6 shows examples of the results of performing these alignment tests in our laboratory.

This figure shows that the center of the target of interest deviates by no more than a radius of $\sim 5 \mathrm{~mm}$ from the center of the target of interest at a height of $632 \mathrm{~mm}$ and no more than $10 \mathrm{~mm}$ at a height of $732 \mathrm{~mm}$. These radii, the FoV of the spectrometers, which is determined by the choice of fore-optic, the height above the ground, and the view geometry on the hemisphere, determine the total area sampled by the spectrometer. The amount of offset in the alignment of the optical axis of the FoV with respect to the center of the target of interest can have a drastic impact on the total extent of the surface that will be included in the directional reflectance measurements. For example, if a 5-deg fore-optic attachment is used for the downward-looking spectrometer of the GRIT-T system and the distance from the target surface to the sensor at nadir is $732 \mathrm{~mm}$, then it can be shown through basic trigonometry that the sampled area when the fore-optic is oriented at the nadir position is $3208 \mathrm{~mm}^{2}$, and the radius of this sampled area is $32 \mathrm{~mm}$. At extreme zenith angles, the extent of the sampled region increases significantly due to the elongation of the major axis of the sampled, elliptical area by a cosine factor. For a sensor orientation at a zenith angle of $65 \mathrm{deg}$, the observed area is $7588.27 \mathrm{~mm}^{2}$, and the major axis extends $75 \mathrm{~mm}$. During the course of a scan, this elliptically sampled area will be rotated azimuthally by $360 \mathrm{deg}$. This means that an annular region of the target surface will be represented in the directional reflectance measurements at extreme zenith angles that are not included in measurements taken close to the nadir orientation. Ideally, the target surface of interest should be considered homogenous so that this does not become an issue. However, geophysical parameters, such as roughness, grain size, and moisture content, can potentially vary even on local scales, meaning that the total extent of the sampled annular region covered at oblique angles, but not observed at nadir, should be kept as close as possible to the region observed at nadir. In the scenario mentioned, having an optical alignment that deviates by $50 \mathrm{~mm}$, for example, from the desired center of interest, increases the extent of the sampled area at oblique angles by $~ 180 \%$ when compared to a perfectly aligned system. These types of deviation would be more likely for any system having a fixed measurement plane such as that of the earlier GRIT ${ }^{1}$ goniometer system. The GRIT-T system, on the other hand, achieves only a $27 \%$ increase in sampled area at oblique angles at a deviation corresponding to the maximum uncertainty of the alignment when compared to a perfectly aligned system.

\subsubsection{Accuracy, mobility, and rigidity features}

In order to create a system that was accurate, mobile, and field-portable, virtually all of the GRIT$\mathrm{T}$ components had to be custom-designed. In some ways, since the system was intended to be used in austere coastal and desert environments, ensuring accurate movements became one of the most challenging tasks. The ability to track the same spot on the target within $\pm 10 \mathrm{~mm}$ at any reasonable height while measuring, for example, along the sloped surface of a sand dune, required absolute knowledge of every mechanical motion in the GRIT-T design. To obtain this knowledge, the GRIT-T utilizes an independent absolute encoder for every rotational motion on the system. These encoders ensure that the user knows where the system is positioned and pointed, at all times, including the error due to the mechanical play internal to the gearboxes. Furthermore, the GRIT-T utilizes sealed, ingress-protected components to increase reliability and aid in ensuring that even in the sandiest of environments, the accuracy of the mechanical movements would not be compromised.

While the absolute encoders work well to reference a particular movement to the frame, they do not provide overall knowledge of the system's geolocation. To obtain geolocation, the GRITT utilizes a dual-antenna GPS-enabled IMU (VectorNav VN-300), and an industrial-grade inclinometer (NIST-Traceable Rieker Inclinometer). These two devices, when integrated, provide outstanding geolocation information, timing, roll, pitch, and reliable heading. While many 
Harms et al.: Fully automated laboratory and field-portable goniometer used for performing...

632-mm Target Tracking Alignment Test Results (mm)

(a)

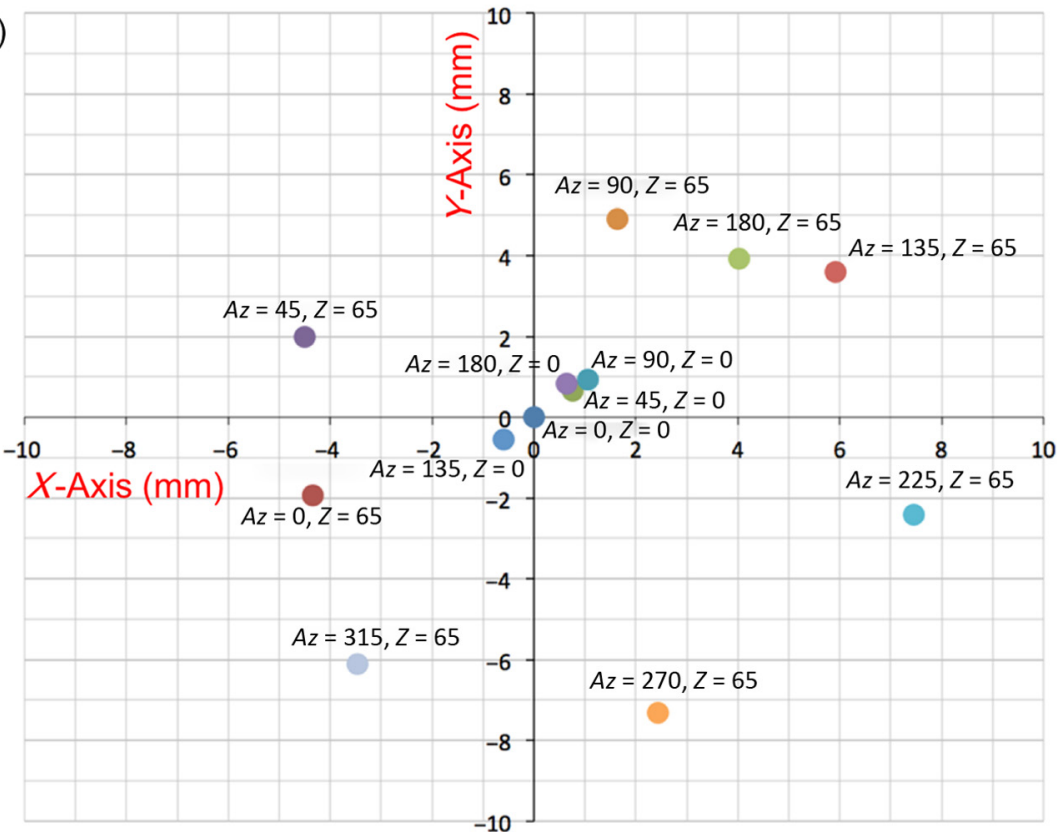

$$
\begin{aligned}
& -A z=0, Z=0 \\
& -A z=0, Z=65 \\
& -A z=45, Z=0 \\
& -A z=45, Z=65 \\
& -A z=90, Z=0 \\
& -A z=90, Z=65 \\
& -A z=135, Z=0 \\
& -A z=135, Z=65 \\
& -A z=180, Z=0 \\
& -A z=180, Z=65 \\
& -A z=225, Z=65 \\
& A z=270, Z=65 \\
& A z=315, Z=65
\end{aligned}
$$

732-mm Target Tracking Alignment Test Results (mm)

(b)
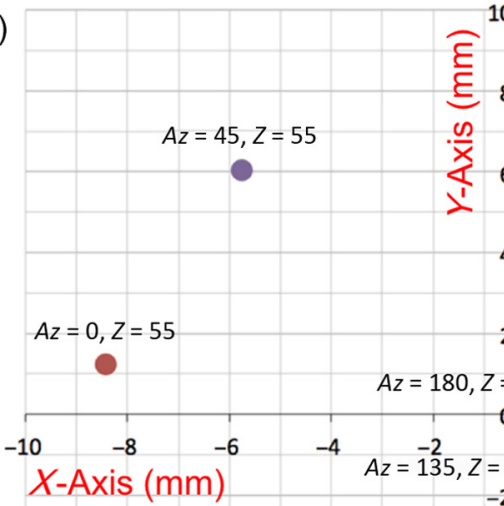

$X$-Axis $(\mathrm{mm})$

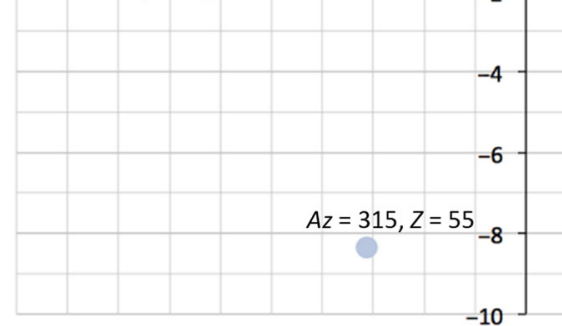

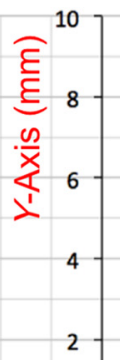

2- $A z=90, Z=0$

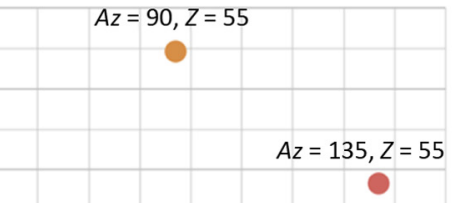

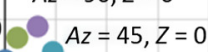
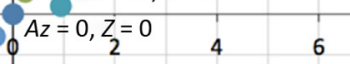

6

$A z=180, Z=55$

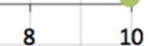

$8-10$

$A z=225, Z=55$

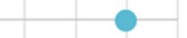
- $A z=0, Z=0$
- $A Z=0, Z=55$
$A z=45, Z=0$
- $A Z=45, Z=55$
- $A Z=90, Z=0$
- $A Z=90, Z=55$
- $A Z=135, Z=0$
- $A Z=135, Z=55$
- $A Z=180, Z=0$
- $A Z=180, Z=55$
- $A Z=225, Z=55$
- $A Z=270, Z=55$
- $A z=315, Z=55$

Fig. 6 The results of performing alignment calibration operations on the GRIT-T system. These images show a coordinate system that is relative to the center of the GRIT-T C-shaped ring. Tests were done for scenarios where the sensor to target distance was (a) $632 \mathrm{~mm}$ and (b) $732 \mathrm{~mm}$. The legend keys show the azimuth and zenith orientation of each recorded sensor orientation of the hemispherical scan pattern.

earlier field-portable goniometers have leveraged GPS for geolocation and timing, the IMU utilized by the GRIT-T has dual GPS antennas that eliminate the need to obtain heading via a standard magnetometer. Instead, the IMU uses two precisely placed, high-gain GPS antennas at a well-known baseline: the GPS antennas sit atop the two actuators on each end of the GRIT-T azimuth ring. This ensures that the heading of GRIT-T is known to within 0.3 deg despite the presence of electronics and stepper motors nearby that would make magnetometer measurements 
unreliable. Thus, due to the on-board GPS/IMU, the GRIT-T provides the user with excellent knowledge of system geolocation as well as solar position. The GPS antennas (and their ground planes) can be seen in Fig. 1 on top of the actuators that are positioned at the two ends of the ring.

Also shown in Fig. 1 are the actuators that allow the GRIT-T to accommodate a wide variety of terrain. These three sealed devices are used to ensure that the system is level before a scan begins. Controlled by one of the embedded computers, these actuators are used to automatically level the system with the aid of the industrial-grade inclinometer. This allows the system to be leveled before the start of a scan and to report the roll and pitch to within \pm 0.1 degree throughout the course of the scan; this information is included in the output accompanying each spectral measurement from the two on-board spectrometers, along with the current state of all other on-board devices. Since the roll and pitch are monitored throughout the course of the scan, the GRIT-T postprocessing routines can incorporate information regarding the leveling of the system. With each movement of the system, the GRIT-T provides the user with the "true" view angle based on the actual position of the sensor reported by the absolute encoders, in addition to the commanded sensor orientations, both of which are reported to the user in the GUI. This automated system leveling, combined with the target-plane tracking, allows the system to measure the same spot of the target on a sloped surface, without compromising angular accuracy, as shown in Fig. 7.

A key factor in achieving mobility for this type of system is ensuring that it is lightweight enough to be easily carried by our team members. The GRIT-T was designed to be carried by just two people under normal circumstances on level terrain and by just three people under unusual circumstances on uneven terrain. To ensure a design that was lightweight, yet rigid enough to provide high accuracy, many of the GRIT-T components were refined using finite-element analysis and then custom-machined from aluminum. Due to the C-shaped design, the ring was one of the most challenging components to design and manufacture and was ultimately created from three separate aluminum slabs. This allowed for a structural layer, a geared layer, and a guide rail layer that were aligned with guide pins and secured using M12 bolts. Even though the ring required a large, solid cross section (roughly $60 \mathrm{~mm} \times 50 \mathrm{~mm}$ ) to reduce torsional rotation when the system was elevated, the aluminum construction ensured that the ring remained

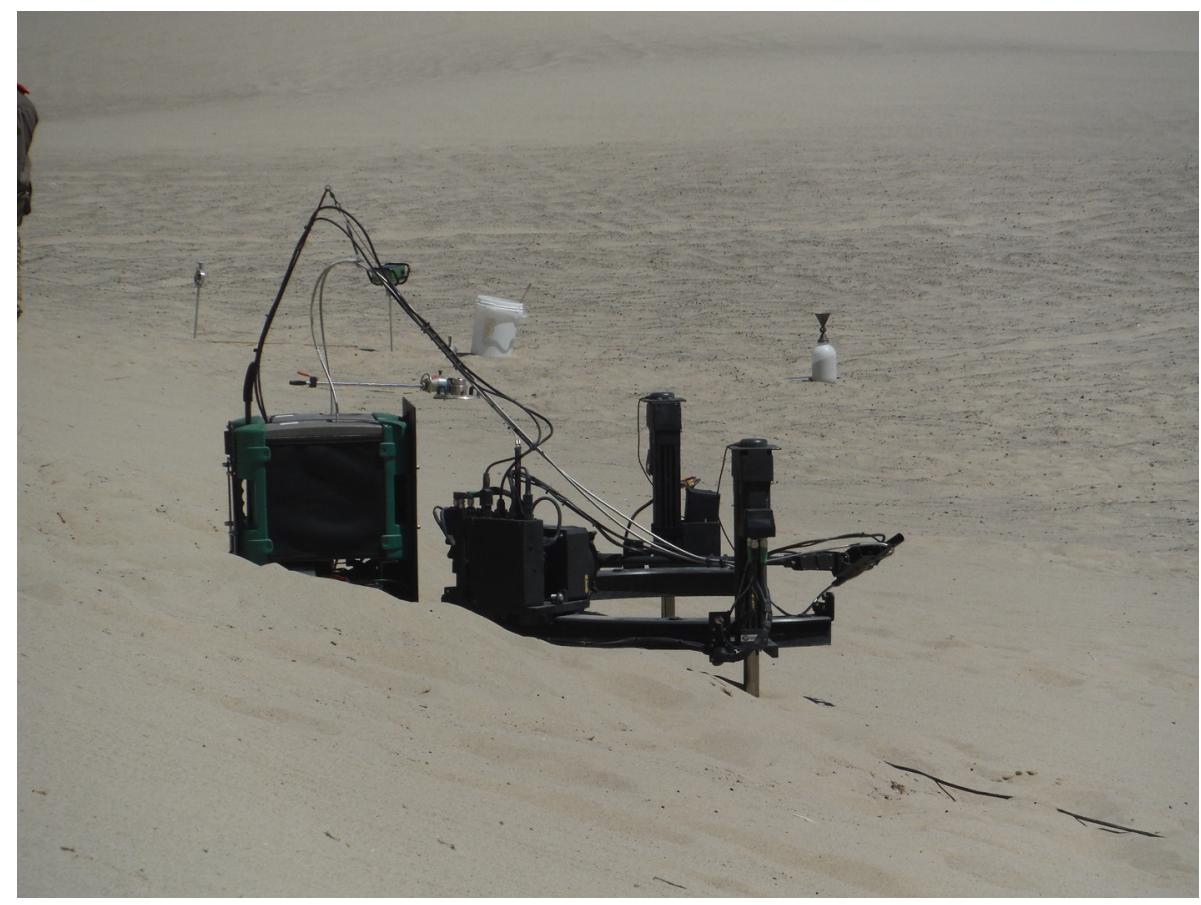

Fig. 7 The GRIT-T as it measures the radiance reflected from a sloped sand surface. Note that the goniometric system is leveled and still able to track the same spot on the target. Also note that, on a sloped surface, the maximum zenith angle that can be reached is reduced, since the distance from the sensor to the target is enlarged. 
reasonably lightweight $(19 \mathrm{~kg})$. In order to allow for use in coastal environments with salt spray, the GRIT-T ring was black anodized and then painted with Krylon 1602 ultraflat black paint to reduce secondary scatter from the instrument frame. The components that contributed to the alignment of the carriage, i.e., the guide rail and the associated bearings, were either black anodized for wear protection or covered to minimize optical impact. All of the other components on the GRIT-T, with the exception of the spectrometers, are rated to at least the IP 54 standard for ingress of dust or water. The IP 54 standard indicates that equipment can operate in environments where water may be splashed on the component and ingress of dust is limited to such a degree that it does not limit operation of the instrument over multiple hours. ${ }^{18}$

\subsection{Minimized Obscuration}

An important feature incorporated into the GRIT-T design was the minimization of obscuration due to self-shading. At small phase angles, i.e., at angles where the sun or other light source is behind the sensor, most systems cast a large, unwanted shadow onto the target plane due to the sensor carriage, and a lot of valuable information from the HCRF or BCRF is lost. In order to measure as much of the HCRF or BCRF as possible, the cross section of the sensor head needs to be minimized. Therefore, the design of the GRIT-T sensor carriage utilized a narrow pointing arm and a narrow pointing head that only cast shadows onto the target plane when the system is measuring close to the opposition direction. The sizes of these components were minimized while still including motors, gearboxes, and a laser range finder required to enable other features. An image of the pointing head can be seen in Fig. 3. To reduce shadows from overhead structures, the GRIT-T was designed with a C-shaped (i.e., $220 \mathrm{deg}$ ) ring that required no other structure to ensure rigidity. Furthermore, the ring was positioned low to the ground to ensure that it did not obscure the downwelling skylight. The remaining structure was then designed to be as short as possible while still meeting other requirements. The only other notable obscurations are from the spectrometers, the two leveling actuators at the ends of the ring, and the pointing arm control hardware. Using these design features, the open side of the ring is intended to be aimed toward the Sun, or, in a laboratory setting, the illumination source. This ensures that the number of points on the spherical grid that are impacted by self-shading from the direct illumination depend only on the width of the pointing head. With this in mind, the width of the pointing head was minimized to $30 \mathrm{~mm}$. Typically, this allows for clean measurements in the solar principal plane for all points on the spherical grid except those with phase angles $\leq 5 \mathrm{deg}$.

\subsection{Digital Elevation Model}

As a result of the GRIT-T's ability to simultaneously rotate its pointing head and pointing arm, the system is also capable of maintaining a nadir view of the measurement plane throughout the entire hemisphere of its range of motion. By combining this capability with the use of the laser range finder on-board the system, the GRIT-T has a sampling mode to produce a DEM of the region surrounding the target. The effort to develop a DEM was motivated by the fact that surface topography on the scale of millimeters plays a significant role in the interaction of light with natural surfaces. ${ }^{19}$ Figure 8 shows an example of a DEM that was measured using the GRIT-T. Here, the sediment was collected during a field experiment in the Nevada desert and dried prior to being used in laboratory measurements. These data are then postprocessed and output as a point-cloud, and metrics of roughness, such as mean slope angle, random roughness, and sill variance, are calculated for use in photometric modeling.

\section{Results}

The GRIT-T was tested in the laboratory at the Rochester Institute of Technology and tested in the field in the Nevada desert. In order to show an example of a laboratory-based BCRF measurement, we present a characterization of a homogeneous sediment that was collected from the Algodones Sand Dunes System, Brawley, California. In the laboratory, we manipulated the orientation of a 150-W tungsten-halogen studio lamp to be oriented at roughly 6-deg azimuth and 


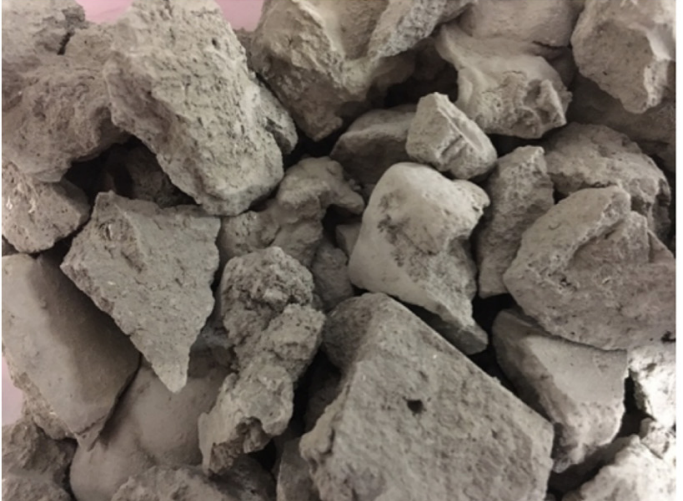

(a)

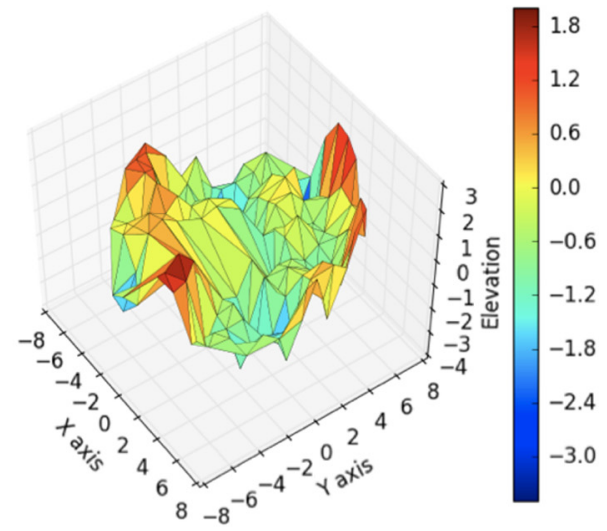

(b)

Fig. 8 (a) Sediment collected in the Nevada desert and (b) a DEM of the sediment using the GRIT$\mathrm{T}$. The DEM has units of centimeters along the $x-, y-$, and $z$-axes. Note that the rocky appearance of the sediment surface is captured, allowing metrics of roughness to be calculated for analysis.

50-deg zenith. Likewise, in order to show an example of a field-based HCRF measurement, we present a characterization of an inhomogeneous sediment that was measured in an austere environment in the Nevada desert in which the sun was oriented at roughly 30-deg zenith and -30- deg azimuth. Sky conditions throughout the course of this field measurement were mostly clear with few clouds. The sediment collected from the Algodones Sand Dunes System contains quartz, feldspar, rock fragments, and heavy minerals (e.g., magnetite, epidote, zircon, biotite, garnet, tourmaline, and hornblende). The quartz is $70 \%$ to $80 \%$ of the composition, the feldspar is $10 \%$ to $15 \%$, the rock fragments are $5 \%$ to $15 \%$, and the heavy minerals are $<1 \%{ }^{20}$ The sediment measured in the Nevada desert contains nonspecific fine-clay soil, river rock, and sand.

For the laboratory-based BCRF measurements, the GRIT-T used a scan pattern with 169 view angle positions on the hemisphere, and for the field-based HCRF measurements, the GRIT-T used a scan pattern with 61 view angle positions on the hemisphere. The scan patterns are shown in Fig. 9. In the laboratory, we have the ability to measure the radiance reflected from a surface under controlled environmental conditions, so the scan patterns can be as dense and, therefore, as long as desired. In the field, however, we do not have this luxury. To minimize the variations due

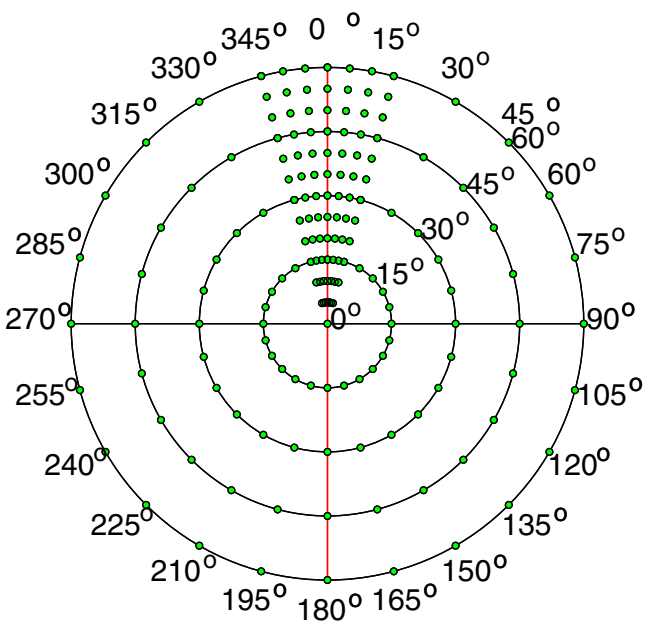

(a)

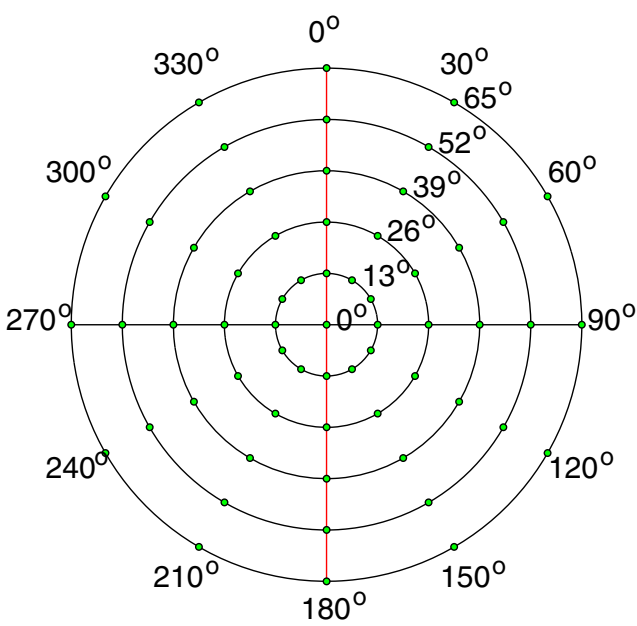

(b)

Fig. 9 Examples of the custom-designed scan patterns for the GRIT-T in (a) a laboratory setting and (b) in a field setting. The sampled orientations are shown by the green points along the polar plot. In the laboratory example, the scan pattern has a higher measuring density around the backscattered region of the solar principal plane, and as a result, the scan pattern has 169 view angle positions. In a field example, the scan pattern has 61 view angle positions. 
(a)

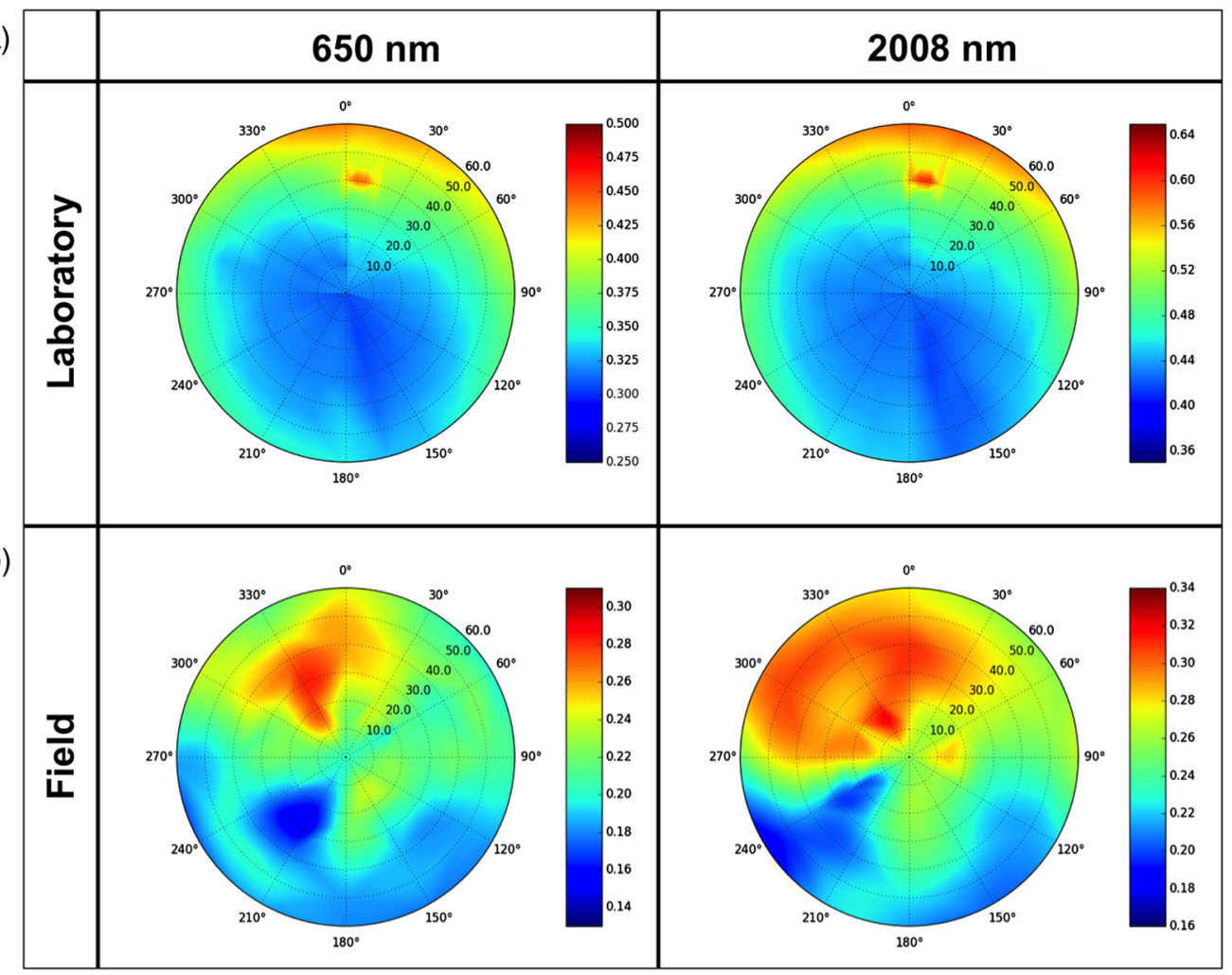

Fig. 10 (a) BCRF data of the sediment collected from the Algodones Sand Dunes System, Brawley, California, and measured in the laboratory at RIT and (b) HCRF data of the sediment measured in the Nevada desert. BCRF and HCRF are both dimensionless quantities, which are easily seen from the definition in Eq. (1).

to the atmosphere, the time of day, and the temporal changes in illumination, we investigated the trade space between the density of the scan pattern and the collection time of the scan pattern. In the field, the selected scan pattern allowed us to measure the radiance reflected from a surface in 35 min while tasking the spectrometers to capture 80 spectral measurements per view angle to ensure adequate signal-to-noise in the short-wave infrared. In addition to these measurements, the radiance reflected from a Spectralon ${ }^{\mathrm{TM}}$ reference panel was measured at the beginning and end of each scan pattern. Here, the Spectralon ${ }^{\mathrm{TM}}$ reference panel was measured at the nadir view angle position in order to compensate for changing illumination conditions due to cloud cover. Moreover, the radiance onto the surface was measured simultaneously with the third spectroradiometer. The on-board ASD FR4 spectroradiometers record 2151 spectral channels in 1-nm intervals in the spectral range between 350 and $2500 \mathrm{~nm}$. The examples shown in Fig. 10 are for two of these spectral channels. Note that the laboratory data show a much higher symmetry due to the fact that the collimated light source remains stationary throughout the scan, whereas the field data show a lower symmetry due to the fact that the sun constantly moves throughout the course of the scan. It is also worth noting that adjacency effects due to roaming cloud cover can cause asymmetries in the collection of field data. Figure 10 shows the visualization of the BCRF data and the HCRF data in 2-D polar plots for two wavelengths-one in the visible at a wavelength of $650 \mathrm{~nm}$ and one in the short-wave infrared at a wavelength of $2008 \mathrm{~nm}$.

\section{Conclusions}

The availability of accurate and precise multiangular reflectance measurements is important for the improvement of radiative transfer models and for the vicarious calibration of remote sensing platforms. For this purpose, a new, fully automated laboratory and field-portable goniometer has been developed and is described in this paper. The GRIT-T, completed in May 2016, 
is a goniometric system that incorporates a dual-view capability with two ASD FieldSpec 4 Hi-Res spectroradiometers, an integrated software for full automation, a set of actuators for automated system leveling, an ability to perform "target-plane" tracking, a minimized obscuration due to self-shading, a DEM of the target surface, and a few other accuracy, mobility, and rigidity features. These combined features have created one of the most advanced and accurate fieldportable goniometers to date that measures the HCRF (field) or BCRF (lab) of a target with a pointing accuracy of $\pm 0.2 \mathrm{deg}$ in both azimuth and zenith. The system is equipped with two spectrometers, a dual-antenna GPS, an industrial-grade inclinometer, a laser range finder, and a miniature FoV camera. The postprocessing routines inherent to the design provide a reliable method for measuring the angular-dependent incoming illumination and the temporal changes in illumination. The GRIT-T has been tested in the laboratory and the field and has proven its value to characterize the BCRF and HCRF of various soils and low-growing vegetation-even in austere coastal and desert environments.

\section{References}

1. C. M. Bachmann et al., "Improved modeling of multiple scattering in hyperspectral BRDF of coastal sediments observed using the goniometer of the Rochester Institute of Technology (GRIT)," Proc. SPIE 9611, 96110J (2015).

2. F. Nicodemus et al., "Geometrical considerations and nomenclature for reflectance," National Bureau of Standards Report, NBS MN-160, U.S. Government Printing Office, Washington, D.C. (1977).

3. G. Schaepman-Strub et al., "Reflectance quantities in optical remote sensing-definitions and case studies," Remote Sens. Environ. 103, 27-42 (2006).

4. K. Z. Doctor et al., "Wavelength dependence of the bidirectional reflectance distribution function (BRDF) of beach sands," Appl. Opt. 54(31), F243-F255 (2015).

5. B. Hapke, Theory of Reflectance and Emittance Spectroscopy, Cambridge University Press, New York, New York (2012).

6. C. M. Bachmann et al., "Flexible field goniometer system: the goniometer for outdoor portable hyperspectral earth reflectance," J. Appl. Remote Sens. 10(3), 036012 (2016).

7. C. M. Bachmann et al., "Modeling geophysical properties of the Algodones Dunes from field and laboratory hyperspectral goniometer measurements using GRIT and comparison with G-LiHT imagery," Proc. SPIE 9972, 99720K (2016).

8. C. J. Bruegge et al., "Parabola III: a sphere-scanning radiometer for field determination of surface anisotropic reflectance functions," Remote Sens. Rev. 19(1-4), 75-94 (2000).

9. J. D. Harms et al., "A next generation field-portable goniometer system," Proc. SPIE 9840, 98400J (2016).

10. J. Schopfer et al., "Dual field-of-view goniometer system FIGOS," in 10th Int. Symp. on Physical Measurements and Spectral Signatures in Remote Sensing (ISPMSRS), M. Schaepman et al., Eds., pp. 493-498 (2007).

11. T. H. Painter, B. Paden, and J. Dozier, "Automated spectro-goniometer: a spherical robot for the field measurement of the directional reflectance of snow," Rev. Sci. Instrum. 74, 51795188 (2003).

12. J. Suomalainen et al., "Finnish Geodetic Institute field goniospectrometer (FIGIFIGO): a device for polarized multiangular reflectance measurements," in IEEE Int. Geoscience and Remote Sensing Symp. (IGARSS '08) (2008).

13. P. P. Roosjen et al., "A laboratory goniometer system for measuring reflectance and emittance anisotropy," Sensors 12(12), 17358-17371 (2012).

14. J. Schopfer et al., "The improved dual-view field goniometer system FIGOS," Sensors 8(8), 5120-5140 (2008).

15. A. Hueni et al., "Visualisation, processing and storage of spectrodirectional data based on the spectral database SPECCHIO," in IEEE Int. Geoscience and Remote Sensing Symp. (IGARSS 2009), Vol. 1, pp. I-84-I-87 (2009).

16. C. M. Bachmann et al., "A dual-spectrometer approach to reflectance measurements under sub-optimal sky conditions," Opt. Express 20(8), 8959-8973 (2012). 
17. ASD Incorporated, "Fieldspec 4 Hi-Res: high resolution spectroradiometer," 2015, http:// www.asdi.com/products/fieldspec-spectroradiometers/fieldspec-4-hi-res (24 November 2015).

18. NEMA, "NE IP reference chart 0614: IP (ingress protections) rating guide," 2017, https:// www.nemaenclosures.com/media/catalog/category/ratings/NE_IP_Reference_Chart_0614. pdf (28 August 2017).

19. B. Hapke, "Bidirectional reflectance spectroscopy: 3. correction for macroscopic roughness," Icarus 59(1), 41-59 (1984).

20. R. S. U. Smith et al., "Mineral resources of the North Algodones Dunes wilderness study area (CDCA-360), Imperial County, California," No. USGS-OFR-84-630, U.S. Department of the Interior Geological Survey, California (1984).

Justin D. Harms received his BS degree in electrical engineering from Southern Illinois University, Edwardsville, in 2009, his MS degree in electrical engineering from the University of New Mexico in 2012, and his PhD in imaging science from the Rochester Institute of Technology in 2016. He has worked on a variety of defense programs and is primarily focused on photonics and related systems.

Charles M. Bachmann received his AB degree from Princeton University in 1984, his ScM degree in 1986, and his $\mathrm{PhD}$ in 1990 from Brown University. He was a research physicist at the Naval Research Laboratory from 1990 to 2013, serving as head from 1993 to 2013 of the Coastal Science and Interpretation Section in the Remote Sensing Division. He has been the Frederick and Anna B. Wiedman chair at the RIT Chester F. Carlson Center for Imaging Science since 2013, where he also serves as graduate program coordinator. His research emphasizes hyperspectral remote sensing of coastal and desert environments.

Brittany L. Ambeau received her BS degree in applied mathematics in 2013 and her $\mathrm{PhD}$ in imaging science in 2017 from the Rochester Institute of Technology. She currently works in the defense community as a senior scientist. Her research interests include hyperspectal remote sensing, image chain simulation, and vicarious calibration.

Biographies for the other authors are not available. 